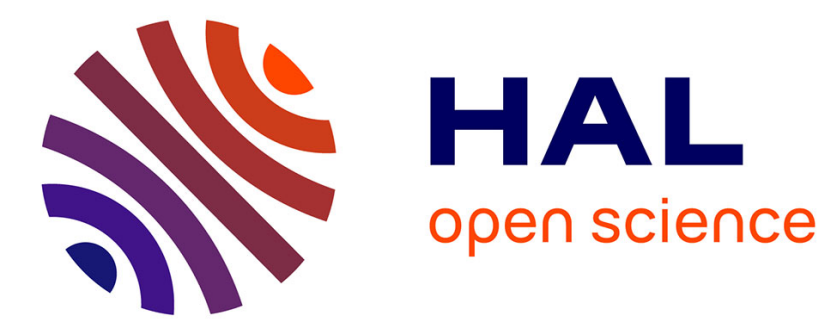

\title{
LES ENTRÉES DE VILLE: UNE AFFAIRE DE COMMERCE OU D'HOSPITALITÉ
}

\author{
Christophe Gibout
}

\section{To cite this version:}

Christophe Gibout. LES ENTRÉES DE VILLE: UNE AFFAIRE DE COMMERCE OU D'HOSPITALITÉ. Les Annales de la Recherche Urbaine, 1998. hal-03017163

\section{HAL Id: hal-03017163 \\ https://hal.science/hal-03017163}

Submitted on 20 Nov 2020

HAL is a multi-disciplinary open access archive for the deposit and dissemination of scientific research documents, whether they are published or not. The documents may come from teaching and research institutions in France or abroad, or from public or private research centers.
L'archive ouverte pluridisciplinaire HAL, est destinée au dépôt et à la diffusion de documents scientifiques de niveau recherche, publiés ou non, émanant des établissements d'enseignement et de recherche français ou étrangers, des laboratoires publics ou privés. 


\section{LES ENTRÉES DE VILLE: UNE AFFAIRE DE COMMERCE OU D'HOSPITALITE}

\begin{abstract}
«Ville: là où s'est écoulée pendant des siècles une vie digne de l'être [...]. Or ce trajet et cette espérance que les lumières avaient assignés à l'homme, la vie moderne l'a vu s'achever dans de nouvelles banlieues et leur cœur vide, qui n'est plus une place publique, une agora, mais une galerie marchande, un centre commercial [...]. Que reste-t-il en effet dans ces grises boutiques de béton armé, autour desquelles on convie les hommes de l'Europe moderne à demeurer, de ces anciennes utopies, de la belle place monumentale des siècles classiques, ornementée, civique, religieuse? Où prendre son essor, sur quoi s'appuyer, sur quel modèle se fonder? Sous les galeries marchandes, devant les vidéoshops, entre les caddies renversés, l'écho d'une boîte roule dans le vent qui fraîchit. »

Danielle Sallenave, Le Don des Morts, Paris, Gallimard, 1991.
\end{abstract}

a vision nostalgique de la ville et de ses franges urbaines, le pessimisme qui sourd de tout ce que nous pouvons ordinairement lire ou entendre, trouvent chez Danielle Sallenave une de leurs plus belles expressions. Les entrées de villes restent encore trop souvent, particulièrement dans le discours distingué, perçues comme des zones de «non-ville», niant toute expression d'un rêve urbain et toute possibilité de sortie d'une crise de la ville dont l'expression esthétique la plus criarde et la plus désolante demeurerait, au-delà des barres et autres cités HLM, ces ouvrages partout identiques, ces bâtiments sans formes, uniformes et empilés les uns près des autres. Entrer en ville, c'est effectivement d'abord franchir une zone plus ou moins vaste où les espaces commerçants s'ajoutent aux surfaces marchandes, dans un agglomérat, souvent disgracieux, de tôles, d'enseignes lumineuses et de cubes dits encore «boîtes à chaussures». Nul ne semble pouvoir aujourd'hui échapper à cette évidente nécessité. De Bordeaux à Reims, de Tours à Montpellier, entrer en ville c'est donc, aujourd'hui, franchir ces «nonlieux» que certains qualifient de prototypiques d'une surmodernité en construction.

Ces nouvelles pratiques périurbaines répondent à de nouvelles pratiques sociales et en créent. Nous pourrions nous risquer à une analyse de ces aménagements urbains en termes de dispositifs de cohésion sociale ${ }^{1}$ et d'incita-

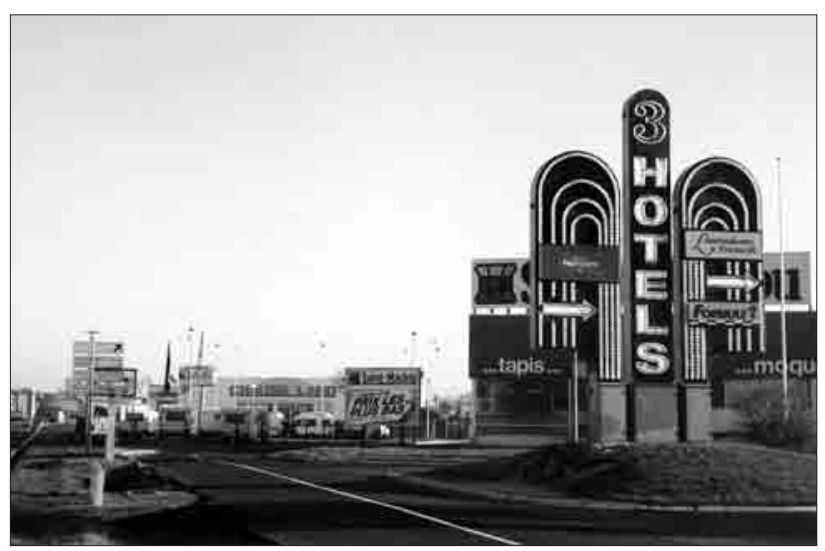

Entre le Futuroscope et la vieille ville de Poitiers.

tion à la dépense. Leur création vise d'abord à accroître la demande de la population. Lui proposer l'entrée dans le vertige de la consommation est également une façon de l'attirer hors de ses véritables problèmes. Les grandes surfaces installées et construites dans nos banlieues apparaîtraient alors comme des dispositifs de contrôle social. En concentrant la masse dans l'espace restreint et circonscrit du supermarché, il serait plus aisé de la connaître, de l'influencer et de la discipliner.

\section{Le nouveau commerce aux portes des villes}

Dès le début de la cinquième République, les banlieues urbaines ont vu surgir, après et auprès des zones d'habitations populaires, des espaces dévolus à la pratique des «courses». Situés aux franges des centres urbains, ils se caractérisaient par l'utilisation de surfaces auparavant vierges de toute construction. Le choix d'implantation était essentiellement orienté vers des surfaces agricoles voisines des axes routiers et à proximité immédiate des consommateurs pressentis, pour lesquels des parkings

1. Nous nous inspirerons pour ce court développement sur des écrits de Michel Foucault, et particulièrement Surveiller et Punir, Naissance de la Prison, Paris, Gallimard, 1975

Les Annales de La Recherche Urbaine $n^{\circ}$ 78, 0180-930-III-98/78/109/5 @ METL 
étaient mis à disposition afin qu'ils trouvent aisément à se garer avant de tenir leurs rôles de clients.

Peu d'égard était accordé à l'environnement de ces nouvelles constructions et à leur allure physique. Les espaces incertains faits de blocs grisâtres, parfois peints aux couleurs des marques des groupes commerciaux, se sont donc posés au milieu de nulle part. Cette première génération de grands magasins généralistes est implantée entre la ville diffuse et la ville centre. Les premiers supermarchés ont jailli de terre au milieu des champs, participant ainsi de l'extension physique de la ville. Protégées derrière le no man's land de parkings, les surfaces marchandes d'entrées de ville étaient alors l'affirmation d'une urbanité conquérante, nouvel eldorado des temps modernes.

La protection sociale qui s'est généralisée a garanti un revenu constant, largement indépendant de la maladie et de l'activité. Dès lors, le revenu, en constante progression, peut être totalement consacré à la consommation. La nécessité de thésauriser pour des lendemains incertains ou une vieillesse inéluctable a disparu. Pour répondre à cette nouvelle demande toujours croissante, les commerçants ont dû adapter leurs circuits de distribution. Une consommation de survie, liée à des revenus précaires, incitait à des achats de petites quantités et au fur et à mesure des rentrées de revenus ${ }^{2}$. Au contraire, un revenu plus ou moins fixe, garanti par les minima sociaux, la croissance économique rapide, l'absence de chômage, concourent à une rationalisation des dépenses, incite à des achats regroupés et plus importants. Pour faire face à ces nouvelles demandes, les distributeurs ont besoin de stocks plus importants. Les problèmes de stockage incitent à une recherche de positionnement dans des lieux dégagés de contraintes spatiales. La périphérie urbaine s'impose donc comme nouveau lieu d'implantation commerciale.

L'afflux de main d'œuvre immigrée, bientôt suivie par les familles, comme la forte natalité endogène ${ }^{3}$ se sont également associés pour accroître la demande générale en biens et services. Mais, c'est sans aucun doute l'avènement des classes moyennes ${ }^{4}$ qui a servi de dynamiseur à la croissance du périurbain commercial. Les nouvelles professions intermédiaires, tels les cadres particulièrement liés au tertiaire, qui avaient des revenus souvent plus élevés, pouvaient plus aisément consommer, singulièrement des biens de consommation non primaire et des produits de consommation symbolique. De nouveaux espaces de distribution devaient donc être créés. Qui plus est, ces nouvelles classes sociales consomment des produits innovants ou nouveaux. Leur équipement progressif en biens lourds (électroménager, véhicule, plus tard hi-fi, vidéo...) a stimulé la demande. Pour y répondre, les distributeurs ont été tentés de proposer ces biens dans les mêmes lieux que ceux de consommation courante. Ainsi sont nés les supermarchés. Espaces de distribution à large diaphragme de distribution, ces magasins étaient forcés d'accroitre leurs surfaces de vente. De telles disponibilités spatiales étaient difficiles à trouver en centre-ville. Les distributeurs ont donc envahi l'immédiate proximité des villes.

Ces territoires périurbains spécialisés dans le commerce découlent enfin d'une croyance commune dans la science et le progrès. La technique est présentée à l'époque comme le moyen de désaliéner l'homme, d'embellir sa vie, de la rendre plus agréable, plus belle, plus rationnelle et moins pénible. Dès lors, les progrès techniques de la période sont immédiatement traduits dans des applications pratiques. Recherchant une maximalisation de l'utilisation de l'espace à moindre coût, les constructions et aménagements de nos périphéries commerciales utilisent les techniques et les matériaux les plus nouveaux. Ce recours aux procédés récents ou contemporains est d'autant plus présent et nécessaire qu'il sert également de vecteur publicitaire pour les distributeurs commerciaux. Faire usage de ces techniques, c'est aussi signifier au consommateur potentiel son appartenance à ce monde moderne en construction.

Au niveau esthétique, cette religion de la modernité a conduit à la recherche d'une esthétique particulière. Arcbouté sur les idées de fonctionnalité et de rationalité, le recours au béton et au métal et la recherche de volumes simples et de surfaces planes sont les deux points les plus caractéristiques de ces constructions. D'où l'occupation des entrées de nos villes par des espaces commerciaux à l'architecture si caractéristique, mêlant économie des investissements et aspect massif des volumes pour rassurer le consommateur. Les grandes avenues rectilignes et les parkings vouent ces espaces à la pratique de l'automobile. La voiture est indispensable à la fréquentation de ces espaces commerciaux périurbains, mais elle y acquiert le caractère d'un référent symbolique de la modernité urbaine et de l'achèvement d'une liberté individuelle de circulation.

\section{La métamorphose anti-urbaine de la périphérie}

Aujourd'hui encore, les entrées de ville sont des lieux de l'expérience consommatrice. Mais elles sont également les révélateurs de nouvelles données sociales. Dans un mouvement «d'exurbanisation» qui n'a cessé de s'accentuer $^{5}$, les hypermarchés, retirés du tissu urbain stricto sensu, se sont dotés d'éléments d'apparence urbaine, gale-

2. Cf. R. Hoggart, La culture du pauvre, (éd. fr.), Paris, Minuit, 1970.

3. De la fin de la guerre à 1972, les taux de natalité et de fécondité français atteignent leurs plus hauts niveaux, entraînant beaucoup plus que le renouvellement de la population.

4. Cf. Henri Mendras, La seconde Révolution Française, 1965-1984, Paris, Gallimard, 1988, rééd. 1994, Folio Poche, 1994.

5. Une estimation récente chiffrait à 36000 hectares, la surface totale, hors Paris, occupée par les corridors commerciaux des périphéries d'agglomérations françaises (Cf. Stéphane Lupieri, "Le Musée des Horreurs de I'Urbanisme Commercial», Alternatives Économiques, mai 1995, p. 44-46). 
ries marchandes ou jardins intérieurs. Ces fragments d'une fausse ville juxtaposés aux échantillons les plus convenus d'une ville plus que moderne (autoroutes reliées au monde, architecture de verre, métal et béton par exemple) ont métamorphosé les entrées de ville.

L'encouragement à une pratique de concurrence accrue entre les différentes surfaces commerciales, tant pour accroître leurs marges que leurs bénéfices et donc leurs chances de survie dans un monde économique caractérisé par la forte mortalité entrepreneuriale, a conduit les entrepreneurs, pour appâter le chaland, l'attirer dans leur propre surface marchande, à s'orienter vers de nouvelles pratiques d'intégration de leurs entreprises dans le paysage urbain. Il importait, effectivement, d'être présent, plus visible et lisible encore. Ces pratiques publicitaires exacerbées ont «défiguré » nos entrées urbaines en les transformant en une suite anarchique, et toujours plus épaisse, de placards et autres enseignes publicitaires ou informatives ${ }^{6}$. A la recherche de marquages d'identité a répondu la profusion de signes distinctifs. Fréquemment, de ce trop-plein symbolique a aussi découlé une perte de sens, de lisibilité et d'esthétique ${ }^{7}$.

Cette éclosion de nouvelles surfaces marchandes s'explique également par une modification des pratiques culturelles de consommation. De fait, la consommation de masse caractéristique des trente glorieuses n'a pas disparu. Cependant, elle a subi une évidente évolution vers des pratiques plus individualisées ou plus microsociales ${ }^{8}$. Dans une société moderne, qualifiée par certains de «postsalariat $»^{9}$, les personnes développent des pratiques de consommation plus individuées ou plus caractéristiques de leurs multiples appartenances sociales. Elles cherchent, au travers de l'acte de consommer à combler un triple désir. D'une part, elles veulent se différencier de leurs semblables, d'autre part, elles souhaitent signifier par leurs achats leurs appartenances sociales, enfin elles entendent disposer de biens et de services qui soient sinon uniques du moins présentant des caractères d'adaptation fidèles à leurs désirs.

Dans des espaces plus restreints, différenciés et différenciants, plus que l'acte d'achat, le fait même de se rendre dans tel magasin signe une appartenance sociale. Ainsi sont apparues les moyennes surfaces et les galeries marchandes, induisant par là même un nouvel urbanisme commercial. En effet, non seulement les bâtis étaient d'envergure plus restreinte, mais surtout, ils devaient participer de la nouvelle symbolique de consommation. Dès lors, les architectes eurent toujours plus recours à des agencements urbains et à des décors qui visaient à habiller le cube cloné de la surface d'antan. Ce masque ${ }^{10}$ rendait ainsi l'espace marchand remarquable, à savoir à la fois repérable dans un environnement proliférant et à même de distinguer ses utilisateurs potentiels.

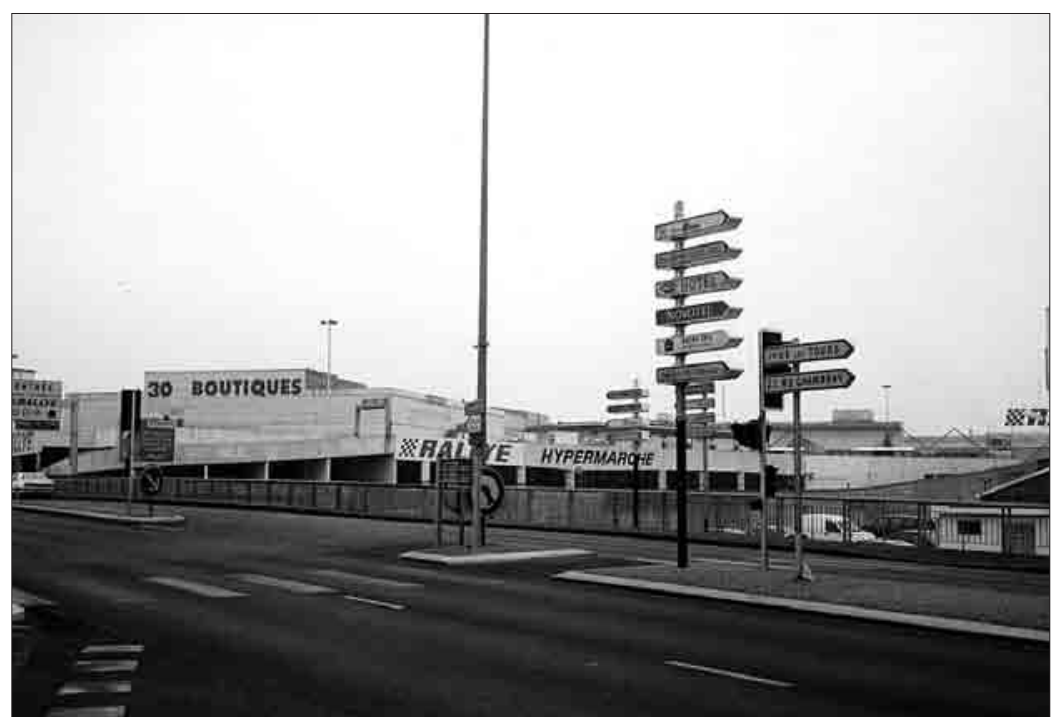

6. En ne comptant que les panneaux 4 fois 3 mètres réglementaires, on estime aujourd'hui que 290000 de ces dispositifs ornent nos villes Pour une vision plus juste de la réalité esthétique des portes de ville, il faudrait y ajouter l'affichage sauvage et les autres techniques de visualisation commerciale.

7. Cette pratique désordonnée a d'ailleurs conduit plusieurs municipalités, à l'exemple de Nîmes, Toulouse ou Brest, à réagir, en s'appuyant sur les mesures facultatives de la loi «Administration du Territoire de la République» de 1992, et à proposer des aménagements publics de ces entrées de villes, lesquels avaient pour objet essentiel de différencier la ville de ces consœurs et de masquer l'enchevêtrement de marques derrière une harmonie de façade propre à rappeler que la cité était originellement le lieu de la civilisation et aussi de la civilité.

8. M. Maffesoli, dans Le temps des tribus: Le déclin de l'individualisme dans les sociétés de masses, Paris, Méridiens, 1988, rééd. Livre de Poche, 1991, constate, dans nos sociétés contemporaines, la disparition des pratiques individualistes les plus manifestes au profit de pratiques sociales propres à des microgroupes éphémères, instables et autorisant de multiples et changeantes appartenances groupales. Nous pensons que ces «tribus» nouvelles sont aussi caractérisées par leurs pratiques culturelles les plus quotidiennes, particulièrement celle de consommer.

9. Robert Rochefort, directeur du CREDOC; (interview EUROPE 1, Janvier 1997. «Nous entrons dans une société de post-salariat... Cette entrée est marquée par le passage progressif de l'individualisme des années 80 à une société centrée sur la personne dans les années 90 . Nous passons du choix de chaussures à pompons rouges car le voisin en possède avec des pompons verts, au choix de chaussures sur mesure dans lesquelles on se sent bien...»»).

10. Au début des années 80 , il s'agissait essentiellement d'ajouts plaqués sur les bâtiments de béton. Puis, progressivement, ces placages de toutes sortes (lumières, bois, calicots, idéogrammes) laissèrent la place à des architectures plus élaborées ou plus propres à identifier. Pour ainsi différencier, les aménageurs jouèrent sur l'apparition de nouvelles techniques de construction et de nouveaux matériaux. La possibilité de construction à structure légère lossature métallique) et d'apports résistants et légers encouragea au développement de tout ce décorum. Aujourd'hui, les responsables commerciaux n'hésitent plus à investir dans des constructions, ou au moins des façades, recourant à des matériaux plus originaux, plus nobles ou plus novateurs, en tout cas permettant des réalisations effectivement et visiblement très différentes de leurs voisines par leur aspect extérieur. 


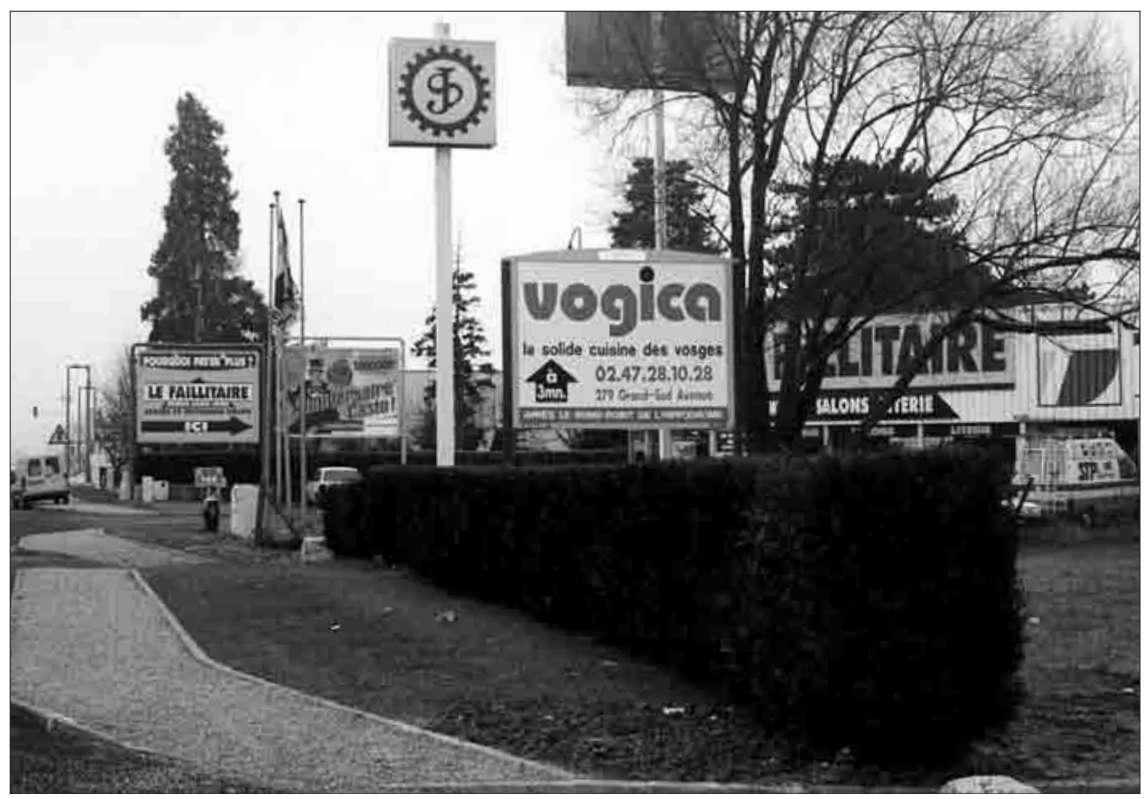

Arborescence publicitaire.

Mais ces modifications des entrées de villes vont également de pair avec une crise prolongée et une modification de la structure sociale du pays. La crise économique permanente depuis le début des années 70 et ses corollaires ont contribué à une modification des habitudes de consommation de la population. La précarité des situations professionnelles a encouragé une thésaurisation et une pratique d'achats économiques. Les faibles revenus d'une part croissante de la nation ont été incités à se tourner vers des commerces à prix réduits, donc à marge faible, donc à gros volumes de ventes. Les supermarchés et autres hypermarchés ont répondu à cette attente.

De la même façon d'autres espaces de vente, magasins de bricolage, d'objets à monter soi-même, de location de matériel, de démarque, se sont installés aux alentours de leurs prédécesseurs ${ }^{11}$. Ils ont investi dans des locaux spartiates, aux formes simples, faits de matériaux ordinaires mais ont recouru, pour s'autopromouvoir, à la panoplie publicitaire la plus complète et la plus accrocheuse pour le regard du public. Il en a résulté une multitude de volumes désordonnés, pauvres en formes, aux couleurs criardes, et séparés les uns des autres par leurs parkings seulement. Il en a découlé une tendance, d'apparence irréversible, à toujours gagner en masse globale et en superficie. Dans ce cas-là, la zone commerciale se distend le long des axes routiers plutôt qu'en profondeur (visibilité oblige!), donnant à la ville une morphologie tentaculaire.

\section{Consommateur en ville ou de ville}

Par ailleurs, les années 80 et 90 se caractérisent aussi par l'apparition de nouvelles classes moyennes, jeunes ou avec enfants, aux aspirations sociales plus élevées que leurs revenus, dont la propension à consommer est très importante. Désirant renouveler plus fréquemment leurs biens, ils recourent à ces nouveaux commerces. Quel que soit leur niveau de ressources, leur pratique de consommation est à la fois forte et symbolique. Leurs achats sont les signes de leur statut social. Ils veulent rogner sur les dépenses non ostentatoires pour privilégier le paraître. Dès lors, ils consomment beaucoup de produits de première nécessité en supermarchés périphériques. Mais, dans le même temps, ils désirent trouver à proximité immédiate des commerces de marque, éventuellement de démarque, qui leur permettent de combler leurs attentes en termes d'imaginaires et de référents sociaux. De cette double exigence, traditionnellement impossible à combler ${ }^{12}$, sont donc nées de nouvelles surfaces commerciales spécialisées et les galeries marchandes.

Les consommateurs ont établi des tactiques visant à contourner partiellement les stratégies des grands groupes commerciaux ${ }^{13}$. Ces techniques de braconnage introduisent une autonomie relative du consommateur ${ }^{14}$ face aux manipulations des comportements et des pratiques initiées par les groupes commerciaux. Parmi ces tactiques, le

11. Ces installations à proximité des centres commerciaux périphériques s'expliquent de plusieurs façons. D'une part, elles visent à prendre le client où il est déjà. D'autre part, le coût plus réduit du terrain, comparativement au centre-ville, et des constructions (moindres exigences architecturales) encourageait à ces localisations. Enfin, la montée en puissance de catégories à revenus moyens ou faibles a conduit ces derniers à s'orienter vers l'auto-production de biens ou du moins le «prêt à monter».

12. En effet, concilier ces deux demandes dans un rite de consommation urbaine traditionnelle est impossible. Jusque dans les années 70 , les commerces bon marché étaient périphériques alors que les magasins diffuseurs de biens et de services non de luxe mais ostentatoires et socialement différentiateurs se trouvaient dans les centres des villes. Il était donc impossible de les pratiquer dans le même temps. Aujourd'hui cette possibilité est offerte, même si un certain type de commerce, et les habitudes sociales qui s'y attachent, recherche les centres urbains.

13. A cet égard, nous pouvons mentionner diverses actions des mouvements associatifs ou de l'extrême-gauche politique, et bien évidemment les dernières révoltes des chômeurs de l'hiver 1997-1998.

14. Si Jean Baudrillard pensait en termes de manipulation mentale des individus dans les sociétés contemporaines, et si Michel Foucault envisageait les «objets» comme des succédanés de panoptiques permettant la manipulation par les comportements et les pratiques, Michel de Certeau (L'Invention du quotidien, vol. 1 "Les Arts de Faire», rééd. Gallimard/Folio Essais, 1990, spécialement p. $\mathrm{XL}$ à XLIII, et p. 50 à 56) propose une autonomie marginale de l'usager face à ces procédures de manipulation. Face à une stratégie de maîtrise de l'espace et de ses habitants, de Certeau note l'existence de possibilité de tactiques, ajustements et détournements des stratégies mises en œuvre par les instances sociales. 
retour à des réalisations individuelles, à des productions personnelles et à domicile ${ }^{15}$. Pour récupérer ces pans entiers de l'économie qui leur échappaient, les stratèges commerciaux ont initié l'établissement des galeries marchandes. Elles répondent alors à un double défi : tenter de dissuader les pratiques d'auto-production par la vente à très bas prix, ou imposer aux personnes non convaincues ou réfractaires des espaces marchands spécialisés dans les biens intermédiaires.

Les mutations culturelles, basée sur l'effervescence des communications, la profusion et la rapidité, qui ont également marqué la société française depuis une vingtaine d'années sont, très concrètement, des déterminants des nouveaux aménagements commerciaux périurbains. La société emportée par une frénésie de communication ${ }^{16}$ se caractérise par une prolifération des messages auxquels le citoyen est confronté. Elle connaît une véritable «épopée de l'œil... Toute réalité est mesurée à sa capacité de se montrer, muant les communications en voyage de $l^{\prime} œ i l{ }^{17}$. L'individu devient un voyeur auquel toujours plus de messages sont destinés.

Dans la confrontation récurrente à des messages tous azimuts, médias de masse souvent disponibles dans les véhicules, panneaux publicitaires, enseignes commerciales aux informations routières, l'annonceur est tenté de faire de la surenchère. Il recourt à une visualisation nominale plus ample, frôlant parfois le démesuré. Ensuite, il cherche à avoir une signalétique qui permette une reconnaissance de la marque par-delà les lieux. Dès lors, les logos et autres insignes s'affichent partout identiques, les bâtiments signent le propriétaire et se détachent de sa ville d'accueil. L'entrée en ville devient partout une suite désordonnée de marques, dont l'originalité la plus grande réside dans la variation différente de la suite d'enseignes que constate le consommateur selon la ville où il entre.
Néanmoins, si les villes ont besoin d'être commodes et de faciliter les relations commerciales, le tourisme et la concurrence nationale et internationale leur imposent une visible magnificence. Les allées de l'entrée en ville ne font pas que faciliter le fonctionnement économique, elles sont des perspectives qui inaugurent la monumentalité de la cité, dont la vocation et la revendication sont plus que jamais d'être, chacune, le centre du monde, l'ouverture à un monde. Plusieurs élus locaux ont donc réfléchi à les domestiquer un peu plus et des mesures législatives ont été prises en ce sens (amendement Dupont). Il existe assurément une potentialité d'évolution esthétique de ces entrées de ville. Après quelques années d'un détour consumériste et mercantile, l'aménagement de l'entrée dans la ville retrouve à la fois sa fonction symbolique de vecteur d'une identité locale et sa signification traditionnelle de porte de la cité. Comment se faire également ouverture vers l'urbanité et fenêtre sur la civilisation?

\section{Christophe Gibout}

15. Il faut, dans ce cadre, différencier deux types de démarches, une volontaire et une de nécessité. Ainsi, d'une manière réfléchie, le recours à ces pratiques peut être décidé comme un moyen de lutter contre la société libérale de consommation. Mais, également, les situations économiques précaires nées d'une crise prolongée peuvent conduire à la nécessité de ces pratiques pour maintenir un niveau d'équipement ou de consommation.

16. Cf. Bernard Miege, La société conquise par la communication, Grenoble, P.U.G., 1990 ; voir également, Erik Neveu, Une société de communication?, (2e éd.), Paris, Montchrétien, 1997.

17. Michel de Certeau, L'invention du quotidien, vol.1, "Les Arts de Faire», rééd. Gallimard/Folio Essais, 1990, p. XLVIII et XLIX.

\footnotetext{
$>$ Christophe Gibout vient de soutenir, à Tours, une thèse en sciences humaines intitulée «Villes et Festivals en Grande-Bretagne et en France». Ancien responsable pédagogique de l'université d'été «langue et civilisation françaises »à Minsk (Bélarus), il est aujourd'hui chargé de cours au département de sociologie de l'Université de Poitiers. Il est membre de l'IPRESCO (Institut Poitevin de Recherches en Sociologie de la Connaissance) et du groupe de recherches «Interface Rural-Urbain » (Département Géographie, Université de Tours).

Ses recherches actuelles, dans les champs des sociologies urbaine, politique ou culturelle, interrogent l'imaginaire politique de l'architecture contemporaine, les temps festivaliers ou, suite à une demande récente, la fréquentation féminine d'un centre
} 